\title{
Combined effects of perceptual grouping cues on object representation: Evidence from motion-induced blindness
}

\author{
Michiaki Shibata, Yousuke KaWachi, ANd Jiro Gyoba \\ Tohoku University, Sendai, Japan
}

\begin{abstract}
We investigated the combined effects of perceptual grouping cues (proximity and contour closure/proximity and orientation similarity) on object representation, using motion-induced blindness, a phenomenon in which salient visual stimuli perceptually disappear when surrounded by moving patterns. We presented as visual targets two stimuli in which a solid square was embedded in an outlined square. Participants reported whether the targets disappeared independently or simultaneously. The results showed that a relatively high proximity cue (with a 0.2-deg separation between the targets) modulated the perceptions of the independent or simultaneous disappearances of targets, regardless of other grouping cues. The contour closure cue modulated these disappearances in the 0.4- to 0.8-deg separations. Finally, the orientation similarity cue began to modulate these disappearances in the 0.6 - to 0.8 -deg separations. We suggest that the separation between the visual stimuli modulates the combined effects of perceptual grouping cues on complete object representation.
\end{abstract}

The visual system groups pieces of visual information into coherent and meaningful objects. Gestalt psychologists investigated this grouping problem in the 1920s. Max Wertheimer (1923), whose works have inspired important past and present vision studies, analyzed the problem based on phenomenological demonstrations and proposed the principles of perceptual grouping (e.g., proximity, similarity, closure, good continuation, and common fate). In recent years, vision scientists have succeeded in investigating perceptual grouping in vision by using more quantitative methods than those used by Gestalt psychologists. For example, Kubovy and colleagues (Kubovy, Holcombe, \& Wagemans, 1998; Kubovy \& Wagemans, 1995) systematically manipulated the separation between dots and the angle between dot strips in multistable dot lattices and asked observers to judge the grouping orientation of the briefly presented dot lattices. They concluded that the dots in a lattice appeared to be attracted to each other in the form of a decreasing exponential function of the separation between them. Furthermore, Elder and Zucker (1993, 1994, 1998) used a visual search task to reveal the role of the closure cue in object perception. They revealed that two-dimensional shape processing was rapid for stimuli with a closed boundary but slow for open stimuli. Thus, perceptual grouping underwent more quantitative studies. Recent investigations have clarified that the presence or absence of visual awareness of objects greatly depends on the strength of perceptual grouping. These investigations used perceptual disappearance phenomena, such as binocular rivalry (Alais \& Blake, 1999; Kovács, Papath- omas, Yang, \& Fehér, 1996), flash-induced perceptual fading (Moradi \& Shimojo, 2004; Vergeer \& van Lier, 2007), motion-induced blindness (Bonneh, Cooperman, \& Sagi, 2001; Mitroff \& Scholl, 2005), and so on. For example, Alais and Blake presented pairs of gratings to one eye and random-dot patches to the other corresponding eye locations in order to produce binocular rivalry. They manipulated the orientations of the gratings and the temporal correlation of contrast modulation of the gratings. They showed that when the two gratings underwent correlated contrast modulation, the gratings appeared together more often than the uncorrelated conditions. They also found that the effects were strongest for collinear gratings. In addition, Vergeer and van Lier found that when the stimuli had the same color or shape, they disappeared simultaneously in flash-induced perceptual fading, in which peripherally presented stimuli perceptually disappear after a flash is presented to the stimuli (Kanai \& Kamitani, 2003). These studies indicated that the contents of visual awareness are determined by aspects of perceptual grouping (Vergeer \& van Lier, 2007).

Most previous studies, including those cited above, have separately investigated the effects of different perceptual grouping cues. A few have systematically investigated more than three perceptual grouping cues by using a quantitative measure of perceptual grouping, which can be defined independently of the observer's verbal reports (e.g., response time; Palmer \& Beck, 2007). Furthermore, remarkably few studies have examined these problems by using the perceptual disappearance phenomenon. How- 
ever, Feldman (2003) mentioned that a single grouping cue cannot define objects, but multiple grouping cues collectively contribute to form objects. Therefore, we need to systematically investigate how the visual system, by using the perceptual disappearance phenomenon, represents partial segments as a complete object on the basis of multiple grouping cues in combination.

Taking both the methodological problem and the research issue together, we investigated the combined effects of multiple grouping cues on object representation. In the present study, we utilized the phenomenon of motion-induced blindness (MIB). According to Bonneh et al. (2001), MIB occurs when salient targets alternately disappear from visual awareness and reappear when they are superimposed on a moving distractor pattern. There are several explanations for target disappearance in MIB. For example, it has been hypothesized that the target disappearance in MIB is caused by attentional competition between the targets and the distractor (Bonneh et al., 2001); by the perceptual filling-in process following a boundary adaptation of the targets (e.g., Hsu, Yeh, \& Kramer, 2004; Hsu, Yeh, \& Kramer, 2006), enhanced by a stereoscopic depth difference between the targets and the distractor pattern; by surface completion of the distractor (Graf, Adams, \& Lages, 2002); and so on. Since MIB and binocular rivalry share some similarities, such as the timing of the perceptual oscillations (Carter \& Pettigrew, 2003), interhemispheric competition may be involved in both MIB and binocular rivalry (Carter \& Pettigrew, 2003; Funk \& Pettigrew, 2003). Caetta, Gorea, and Bonneh (2007) used the signal detection paradigm to show that the target disappearance in MIB is associated with both a sensitivity drop and an upward shift of decisional criteria. These explanations imply that MIB can be caused by different processing mechanisms.

Whereas previous studies explored the mechanisms of MIB, we focused on the vulnerability of target disappearance in MIB to perceptual grouping. In MIB, the targets tend to disappear and reappear simultaneously as complete objects when they are perceptually grouped. On the other hand, when the targets are not grouped, they tend to disappear and reappear independently (Bonneh et al., 2001; Mitroff \& Scholl, 2005). This evidence clearly indicates that the disappearance of targets in MIB is sensitive to the effects of perceptual grouping cues. In MIB, observers can judge the disappearance of targets more easily than in the other perceptual disappearance phenomena, because salient targets completely disappear from one's awareness without any adaptation period, firm fixations, or long training phase. In addition, MIB does not require special equipment (e.g., the use of a mirror stereoscope in a binocular rivalry study) for observation.

Therefore, we used the independent or simultaneous disappearance of the targets as a quantitative measure of the combined effects of perceptual grouping cues on object representation. Additionally, in order to manipulate multiple grouping cues in combination, we formed a geometric configuration of targets in which an outer square contained an inner square in a concentric arrangement. We regarded each of the inner and outer squares as visual tar- gets. By using these stimuli, we were able to create similar stimulus configurations to investigate the combination of proximity/contour closure by varying the relative separations between the stimuli and the contour properties of the stimuli. Likewise, we examined the combination of proximity/orientation similarity cues by varying the relative separations and orientation difference between the stimuli.

In this study, we investigated the combined effects of proximity and contour closure cues (Experiment 1 ) and proximity and orientation similarity cues (Experiment 2) on object representation by using the independent or simultaneous disappearances of the inner and outer targets due to MIB. The results of Experiments 1 and 2 revealed that the proportions of cumulative disappearance duration for independent or simultaneous MIB modes for the full stimulus presentation time (hereafter, "proportion of CDDI" or "proportion of CDDS") and the proportion of relative disappearance duration for simultaneous MIB mode in the total cumulative MIB duration (hereinafter, "the proportion of RDDS") varied depending on the combination of perceptual grouping cues. The proportions of CDDS or RDDS were the largest in the most proximal separation $(0.2 \mathrm{deg})$ between the targets, whereas the proportion of CDDI was the lowest, regardless of other perceptual grouping cues. ${ }^{1}$ With the separation of 0.4 to $0.8 \mathrm{deg}$, contour closure cues had an effect on the proportions of CDDI, CDDS, and RDDS. Moreover, with the separation of 0.6 to $0.8 \mathrm{deg}$, the proportions were affected by orientation similarity cues. The results suggest that the visual system forms a complete object representation by using multiple grouping cues in combination. Moreover, their combined effects are modulated by the separation between the targets.

\section{EXPERIMENT 1}

In Experiment 1, we investigated the combined effects of proximity and contour closure cues on object representation, using stimuli that consisted of inner and outer targets. We experimentally manipulated the spatial separation between the inside edge of the outer target and the outside edge of the inner target (hereafter, "gap distance") by fixing the outer target size and varying the inner target size (Figure 1). Furthermore, we varied the closure level of the outer contour by systematically increasing the length of the horizontal outlines of the outer target. This experiment examined the combination of proximity and contour closure cues by the visual system when representing partial elements as a complete object.

\section{Method \\ Participants. Five students from Tohoku University, age range 22 to 28 years, participated in Experiment 1. Two of them were au- thors of this study. Except for the authors, all the other participants were naive regarding the purpose of the experiment. They all had normal or corrected-to-normal vision and reported no difficulty in the tasks. \\ Stimuli and Apparatus. The stimuli were generated on a Win- dows PC (Precision 390, DELL) using the Cogent software (John} Romaya, Vision Lab, UCL; www.vislab.ucl.ac.uk) for MATLAB 


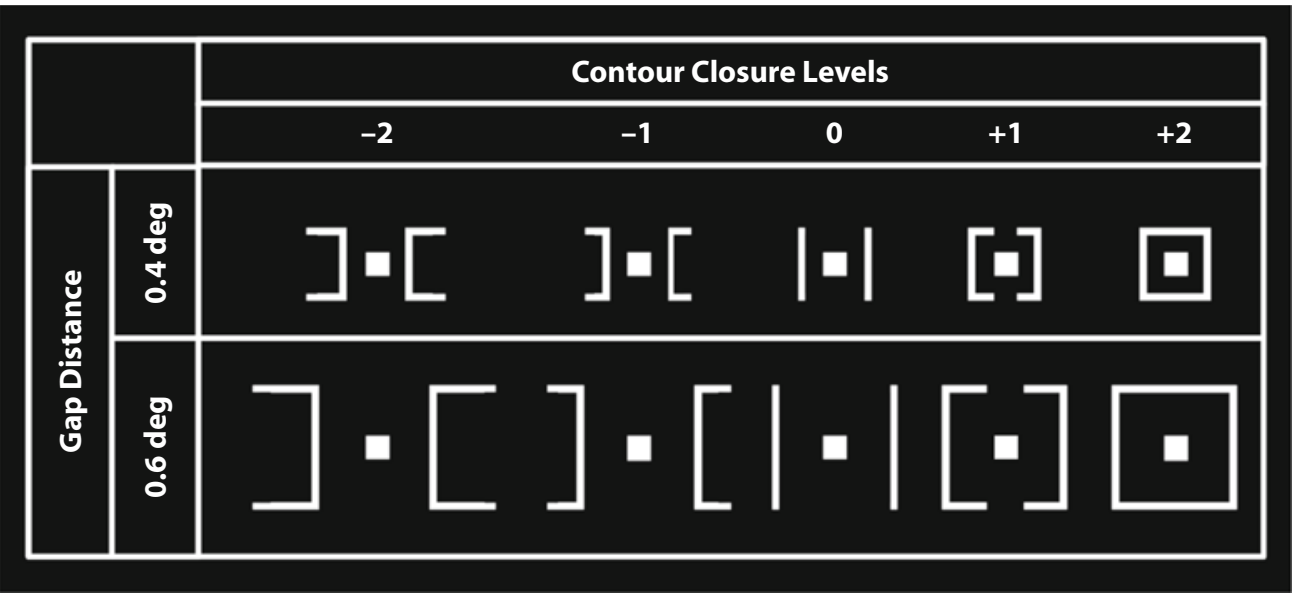

Figure 1. Examples of visual targets used in Experiment 1. The gap distance between the inner and outer squares ranged from 0.2 to $0.8 \mathrm{deg}$. For each gap distance condition, the position of the inner square was always centered, and the horizontal contour of the outer square was gradually lengthened in the outward (or inward) direction, depending on the contour closure levels.

(MathWorks, Inc.) and were presented on a 21-in. CRT display (Sony GDM-F520, 60-Hz refresh rate). The MIB stimulus consisted of a fixation circle, a rotating grid pattern, and two targets (Figure 2); they were presented on a dark background (luminance: $0.55 \mathrm{~cd} / \mathrm{m}^{2}$ ). A red fixation circle (radius, $0.3 \mathrm{deg}$; CIE $x=0.630$, $y=0.352$; luminance, $19.72 \mathrm{~cd} / \mathrm{m}^{2}$ ) was placed in the center of the display. The grid pattern was composed of $7 \times 7$ equally spaced blue cross elements and was approximately $13.7 \times 13.7 \mathrm{deg}$ in size $(\mathrm{CIE}$ $x=0.147, y=0.09$; luminance, $\left.12.12 \mathrm{~cd} / \mathrm{m}^{2}\right)$. Each cross element was $1.5 \mathrm{deg}$, and the separation between the centers of two adjacent crosses was $2.0 \mathrm{deg}$. In order to reduce motion aftereffect, the grid pattern rotated by $180^{\circ} / \mathrm{sec}$ clockwise on the odd-numbered trials and counterclockwise on the even-numbered trials. The two targets were composed of two yellow squares (CIE $x=0.393, y=0.542$; luminance, $81.22 \mathrm{~cd} / \mathrm{m}^{2}$ ). The outer target was an outlined square with a yellow line width of $0.1 \mathrm{deg}$. The inner target was a solid yellow square, centered with respect to the outer target. Both the squares were presented at an eccentricity of $4.1 \mathrm{deg}$ in the upper left part of the fixation circle. In a preliminary observation, we found that a longer MIB was more likely to occur at this point than in any other position. Since increasing the inner target size leads to the deterioration of MIB (Bonneh et al., 2001), the gap distance was manipulated $(0.2,0.4,0.6$, and $0.8 \mathrm{deg})$ by varying the outer target size $(1.08 \times 1.08,1.48 \times 1.48,1.88 \times 1.88$, and $2.28 \times 2.28 \mathrm{deg})$ and keeping the size of the inner target $(0.44 \times 0.44 \mathrm{deg})$ constant. In order to systematically manipulate the contour closure level, we added four horizontal line segments of equal length to both vertical outlines of the outer target in each gap distance condition (Figure 1). Consequently, we set up five contour closure levels $(-2,-1,0,+1$, and +2 ). The positive (or negative) value indicated that the horizontal segments were added to the corners of both vertical outlines toward (or away from) the center of the targets. Each absolute value also indicated the number of the horizontal segments added to each corner of the vertical outlines. Stimuli with a high positive value had a strong contour closure, whereas those in the high negative range had a weak contour closure (Elder \& Zucker, 1993). The grid pattern was not presented within $1.48 \times 1.48$ to $4.2 \times 2.68$ deg spatial ranges around the center of the inner target, and the spatial range depended on each outer target size. These two independent variables were manipulated in a 4 (gap distance) $\times 5$ (contour closure level) factorial design. Participants undertook two experimental blocks consisting of 40 trials each (a total of 80 trials). Each trial lasted for $30 \mathrm{sec}$. Before presenting the experimental blocks, a few practice trials were given to the participants to ensure that they understood the instructions. All the experimental conditions were completely randomized and repeated twice within each block. To prevent fatigue, the task was self-paced, and the participants took short breaks between the blocks.

Procedure. Each participant sat about $57 \mathrm{~cm}$ away from the display in a dimly lit room, head stabilized with a chinrest. The MIB stimulus was presented only after a participant pressed the space bar. Since there are two possible MIB modes in our targets (for motion-induced blindness, Bonneh et al., 2001; for Troxler fading, Hamburger, Prior, Sarris, \& Spillmann, 2006), we asked the participants to judge whether the targets disappeared independently or simultaneously - that is, whether the inner or outer targets disappeared independently ("independent MIB mode"), or whether both targets disappeared simultaneously ("simultaneous MIB mode"). They were asked to press one of two keys to indicate whether the targets disappeared independently or simultaneously, and to release the key when it (or they) reappeared. The duration between pressing and releasing the key was recorded as "the MIB duration." In each trial, the participants were required to maintain fixation on the central circle.

Data analysis. We collected individual observers' responses to the independent and simultaneous MIB modes in all the experimental conditions. The mean proportions of CDDI and CDDS for the full stimulus presentation time $(30 \mathrm{sec})$ and the mean proportion of RDDS in the total cumulative MIB duration, defined as the CDDS divided by the sum of the CDDI and CDDS, were calculated, and we have also calculated the mean disappearance duration of each MIB mode using this data.

\section{Results and Discussion}

The mean proportions of CDDI or CDDS for the full stimulus presentation time $(30 \mathrm{sec})$ of each condition across all participants are shown in Figure 3A. A two-way (gap distance $\times$ contour closure level) repeated measures ANOVA was performed for the mean proportions of CDDI and CDDS, respectively. Overviews of the results of ANOVA are presented in Table 1, in which it can be seen that the main effects of the gap distance and contour closure level, in the proportions of CDDI, were significant. A post hoc analysis with Ryan's method showed that the proportion of CDDI increased as the gap distance between the inner and outer targets increased from 0.2 to $0.8 \mathrm{deg}$ 


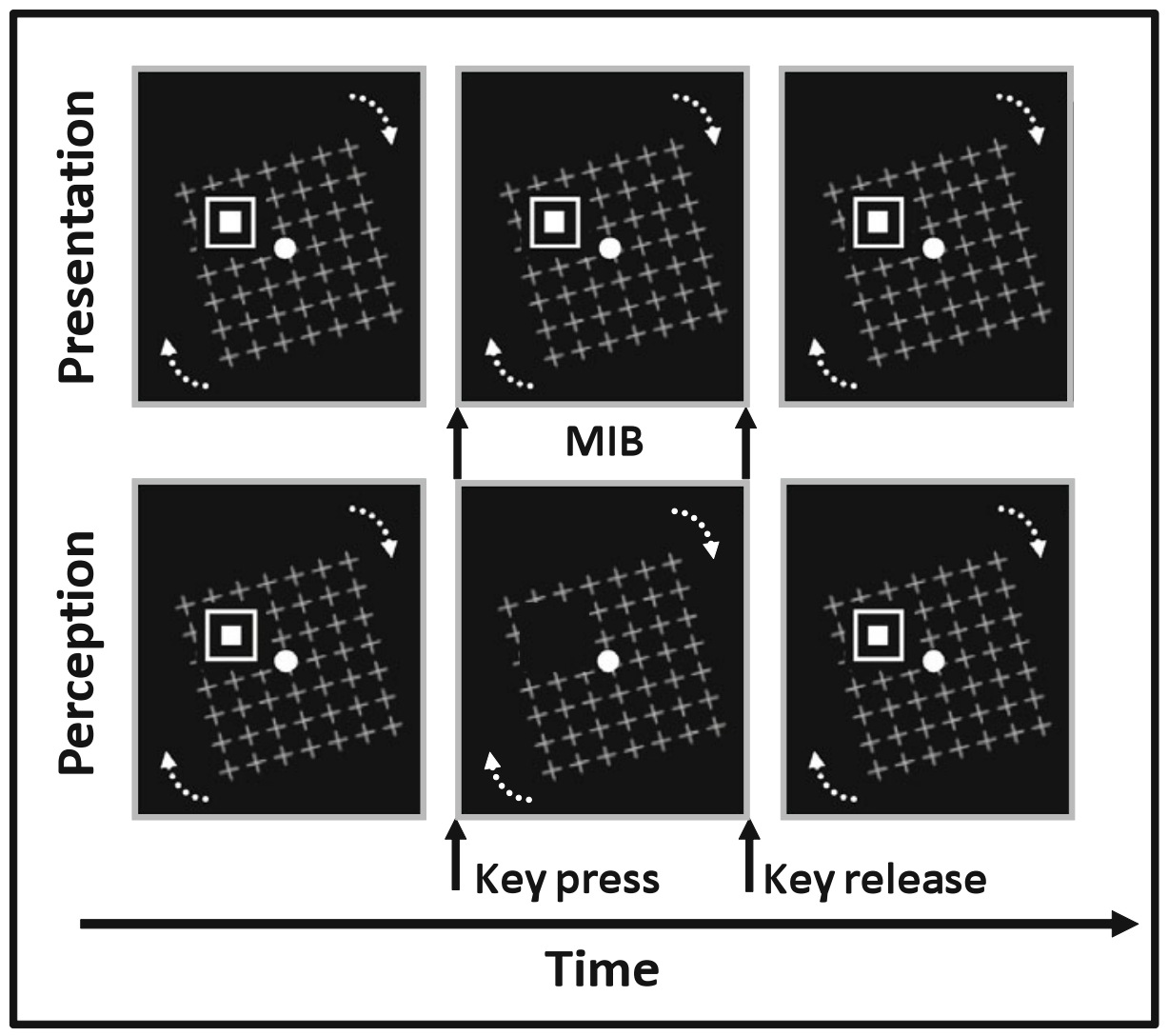

Figure 2. An example of the schematic representation of the MIB stimulus used in this study and the corresponding percept. The participants fixated the cross in the center of the screen while attending to the inner and outer squares in the upper left quadrant. The moving pattern was created from crosses rotated clockwise or counterclockwise at $180 \%$ sec. As soon as one of the targets (or both) perceptually disappeared, the participants were instructed to press an assigned key and hold it down until the target(s) reappeared.

(among all conditions except for that between the 0.2 and $0.4 \mathrm{deg}, p<.05)$. In the main effect of contour closure level, there were also significant differences between the -2 and 0 , between the -2 and +1 , and between the -2 and +2 conditions $(p<.05)$. There was also significant interaction between the gap distance and contour closure level. The simple main effect analysis revealed significant effects of the contour closure level for the $0.4,0.6$, and $0.8 \mathrm{deg}$ conditions of gap distance $[0.4 \mathrm{deg}, F(4,64)=$ 4.64, $p<.005 ; 0.6 \mathrm{deg}, F(4,64)=28.77, p<.001$; $0.8 \mathrm{deg}, F(4,64)=4.82, p<.005]$. A post hoc analysis with Ryan's method showed that the proportion of CDDI decreased as the contour closure level between the inner and outer targets increased from -2 to +2 ( $0.4 \mathrm{deg}$, between the -2 and +2 and between the -1 and +2 conditions, $p<.05 ; 0.6 \mathrm{deg}$, among all the contour closure level conditions except for those between the -2 and -1 and between the +1 and $+2, p<.05 ; 0.8 \mathrm{deg}$, between the -1 and +1 and between the -1 and +2 conditions, $p<.05)$. In the proportion of CDDS, the main effects of the gap distance and contour closure level were significant. A post hoc analysis with Ryan's method showed that the proportion of CDDS decreased as the gap distance between the inner and outer targets increased from 0.2 to $0.8 \mathrm{deg}$ (between all pairs of conditions, $p<.05$ ). In the main effect of contour closure level, there were also significant differences among all the contour closure level conditions, except for those between the -2 and -1 and between the +1 and $+2(p<.05)$ conditions. There was also significant interaction between these two factors. The simple main effect analysis revealed the significant effects of the contour closure level for the 0.4 and $0.6 \mathrm{deg}$ conditions of gap distance $[0.4 \mathrm{deg}, F(4,64)=10.90, p<.001$; $0.6 \mathrm{deg}, F(4,64)=18.13, p<.001]$. A post hoc analysis with Ryan's method showed that the proportion of CDDS increased as the contour closure level between the inner and outer targets increased from -2 to $+2(0.4 \mathrm{deg}$, between the -2 and 0 , between the -2 and +1 , and between the -2 and +2 conditions, $p<.05 ; 0.6 \mathrm{deg}$, between the -2 and +2 , between the -1 and +2 , and between the 0 and +2 conditions, $p<.05)$.

The mean proportion of RDDS in the total cumulative MIB duration of each condition across all participants is shown in Figure 3B. A two-way ANOVA (gap distance $X$ contour closure level) was performed for the mean RDDS percentage data. The ANOVA is presented in Table 1, in which the main effects of gap distance and contour closure level are shown to be significant. A post hoc analysis 
A

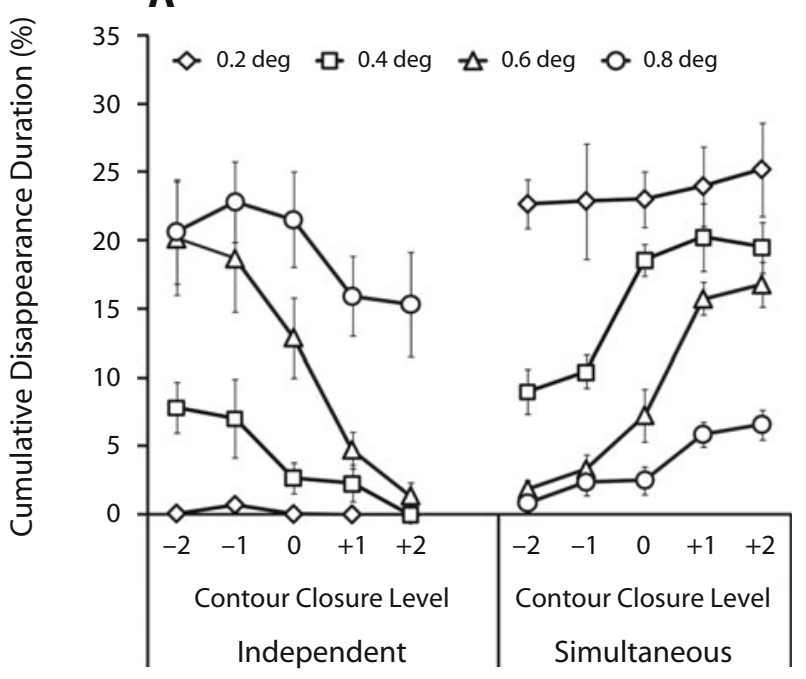

B

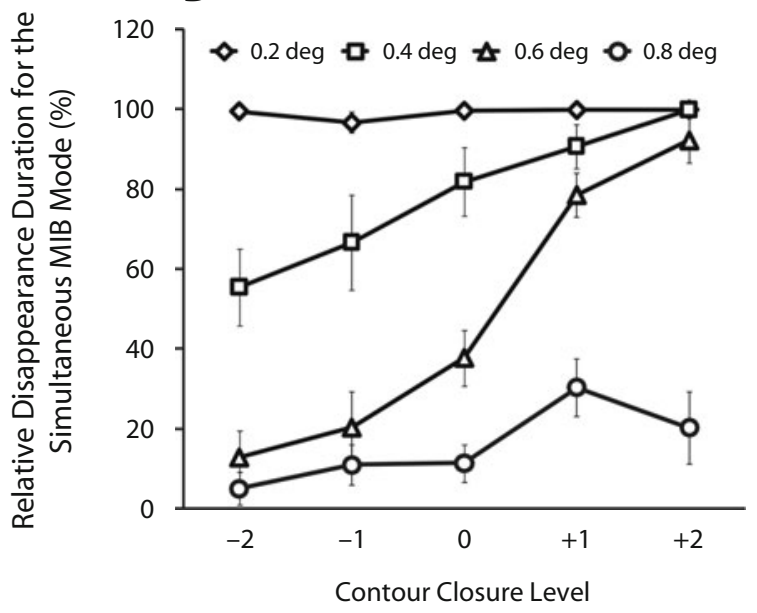

C

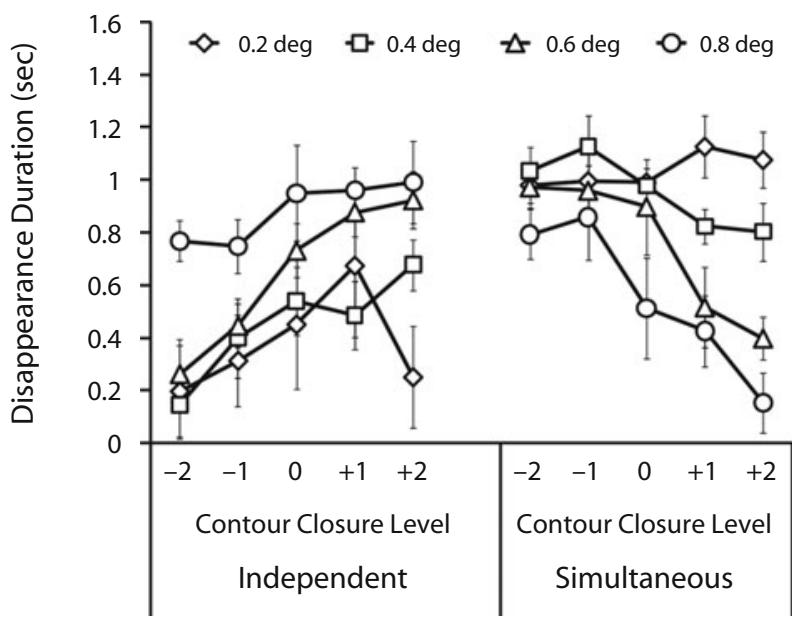

Figure 3. The results of Experiment 1 for each condition. The error bar denotes standard error $(N=5)$. The absence of an error bar means that the SE is too small to be displayed. (A) The $y$-axis indicates the mean proportion of CDDI or of CDDS for the full stimulus presentation time (30 sec). (B) The $y$-axis indicates the mean proportion of RDDS in the total cumulative MIB. For panels A and B, the proportions of CDDS and RDDS increased significantly when the contour closure level was relatively high in the 0.4- to 0.8-deg gap distance conditions. On the other hand, the proportion of CDDI decreased as the contour closure level increased in the 0.4- to 0.8-deg gap distance. (C) The $y$-axis indicates mean disappearance duration of independent or simultaneous MIB modes. For panel C, note that the zero has been used as a missing value. The tendency found in the mean disappearance duration differed from that for the proportions of CDDI, CDDS, and RDDS.

with Ryan's method showed that the proportion of RDDS decreased as the gap distance between the inner and outer targets increased from 0.2 to 0.8 deg (between all pairs of conditions, $p<.05$ ). In the main effect of contour closure level, there were also significant differences among all the contour closure level conditions except for those between the -2 and -1 , between the 0 and 1 , and between the +1 and $+2(p<.05)$. There was a significant interaction between these two factors. The simple main effect of the contour closure level was significant for all conditions of gap distance except for the $0.2 \mathrm{deg}$ condition [ $0.4 \mathrm{deg}$, $F(4,64)=12.26, p<.001 ; 0.6 \mathrm{deg}, F(4,64)=47.52, p<$ $.001 ; 0.8 \mathrm{deg}, F(4,64)=3.70, p<.01]$. A post hoc anal- ysis with Ryan's method showed that the proportion of RDDS increased as the contour closure level between the inner and outer targets increased from -2 to $+2(0.4 \mathrm{deg}$, between the -2 and +2 , between the -1 and +2 , and between the 0 and +2 conditions, $p<.05 ; 0.6 \mathrm{deg}$, among all the contour closure level conditions except for those between the -2 and -1 and between the +1 and +2 conditions, $p<.05 ; 0.8 \mathrm{deg}$, between the -1 and +2 conditions, $p<.05)$.

We performed a two-way (gap distance $\times$ contour closure level) repeated measures ANOVA for the mean disappearance durations of the independent or simultaneous MIB modes, respectively for the full stimulus pre- 
Table 1

ANOVA Summaries for Each Condition in Experiment 1

\begin{tabular}{lcc}
\hline \multicolumn{1}{c}{ Factors } & Independent Significance & Simultaneous Significance \\
\hline & Proportions of CDDI and CDDS \\
Gap distance (GD) & $F(3,12)=28.73, p<.001$ & $F(3,12)=35.88, p<.001$ \\
Contour closure level (CCL) & $F(4,16)=16.77, p<.001$ & $F(4,16)=30.67, p<.001$ \\
GD $\times$ CCL & $F(12,48)=5.87, p<.001$ & $F(12,48)=3.00, p<.005$ \\
& Proportion of RDDS & \\
GD & & $F(3,12)=131.65, p<.001$ \\
CCL & & $F(4,16)=37.08, p<.001$ \\
GD $\times$ CCL & & $F(12,48)=8.94, p<.001$ \\
& & \\
GD & Disappearance Duration & \\
CCL & $F(3,12)=5.80, p<.05$ & $F(3,12)=15.38, p<.001$ \\
GD $\times$ CCL & $F(4,16)=6.68, p<.005$ & $F(4,16)=7.13, p<.005$ \\
& $F(12,48)=1.84, p=.068$ & $F(12,48)=2.32, p<.05$ \\
\hline
\end{tabular}

sentation time. Figure $3 \mathrm{C}$ shows the mean disappearance durations of the independent or simultaneous MIB modes of each condition across all participants. Overviews of the results of ANOVA are presented in Table 1. In the independent MIB mode, the main effects of gap distance and contour closure level were significant. There was no significant interaction between these two factors. A post hoc analysis with Ryan's method showed that the mean disappearance duration of the independent MIB mode increased as the gap distance between the inner and outer targets increased from 0.2 to 0.8 deg (between the 0.2 and 0.8 -deg conditions and between the $0.4-$ and 0.8 -deg conditions, $p<.05$ ). In the main effect of contour closure level, there were also significant differences between the -2 and 0 , between the -2 and +1 , and between the -2 and +2 conditions $(p<.05)$. In the simultaneous MIB mode, the main effect of the gap distance and contour closure level was significant. A post hoc analysis with Ryan's method showed that the mean disappearance duration of the simultaneous MIB mode decreased as the gap distance between the inner and outer targets increased from 0.2 to 0.8 deg (between all pairs of conditions except for those between 0.2 and $0.4 \mathrm{deg}$ and between 0.6 and $0.8 \mathrm{deg}, p<.05$ ). In the main effect of contour closure level, there were also significant differences between the -2 and +1 and between the -2 and +2 conditions $(p<.05)$. There was also significant interaction between these two factors. The simple main effect analysis revealed significant effects of the contour closure level for the 0.6- and 0.8-deg conditions of gap distance [0.6 deg, $F(4,64)=6.02, p<.001 ; 0.8 \mathrm{deg}, F(4,64)=6.76, p<$ $.001]$. A post hoc analysis with Ryan's method showed that the mean disappearance duration of the simultaneous MIB mode decreased as the contour closure level between the inner and outer targets increased from -2 to +2 ( 0.6 deg, between the -2 and +2 , between the -1 and +2 , and between the 0 and +2 conditions, $p<.05$; $0.8 \mathrm{deg}$, between the -2 and +2 and between the -1 and +2 conditions, $p<.05$ ).

The results of Experiment 1 showed that as the gap distance increased from $0.2 \mathrm{deg}$ to $0.8 \mathrm{deg}$, the proportion of CDDI gradually ascended, whereas the proportions of CDDS and RDDS gradually attenuated. Specifically, the relatively high proximity condition $(0.2 \mathrm{deg}$ of gap distance) produced the largest proportions of CDDS and RDDS, regardless of the contour closure cue. Meanwhile, the proportion of CDDI was the lowest in the $0.2 \mathrm{deg}$ of gap distance, also regardless of the contour closure cue. As the contour closure levels increased, the proportion of CDDS became larger in the 0.4 to 0.6 deg of gap distance conditions, whereas the proportion of CDDI gradually decreased in the 0.4 to 0.8 deg of gap distance conditions. In addition, the proportion of RDDS increased as the contour closure levels increased in the 0.4 to $0.8 \mathrm{deg}$ of gap distance conditions. However, the effects for the mean disappearance duration were not always consistent with those for other dependent variables (proportions of CDDI, CDDS, and RDDS). It seems insufficient to judge whether the mean disappearance duration is sensitive to the combination effect of perceptual grouping only from the results of Experiment 1. Therefore, we have discussed the mean disappearance duration in more detail in the Results and Discussion section of Experiment 2, taking into consideration the results of Experiments 1 and 2 together. Overall, the results suggest that when visual stimuli are presented with a gap distance from 0.4 to $0.8 \mathrm{deg}$, the contour closure cues affect object representation. In representing the targets as a complete object, the visual system is not merely restricted by the proximity cue; rather, the proximity cue operates in cooperation with the contour closure cue.

\section{EXPERIMENT 2}

The results of Experiment 1 showed that the proportions of CDDI, CDDS, and RDDS in MIB varied depending on the combination of proximity and contour closure cues. In Experiment 2, we focused on the combination of proximity and orientation similarity cues and investigated how they affected object representation by manipulating the gap distance and orientation difference between the targets.

\footnotetext{
Method

Participants. The same participants completed Experiments 1 and 2 .
} 
Stimuli and Apparatus. The stimuli, apparatus, and procedure were also identical, with the following exceptions.

Procedure. In Experiment 2, in addition to manipulation of the gap distance between the inner and outer targets, we manipulated the orientation difference between them by rotating the outer square from $0^{\circ}$ to $45^{\circ}$, instead of manipulating the contour closure levels. This manipulation can be assumed to decrease orientation similarity (Nelson \& Palmer, 2001). Consequently, four orientation difference conditions were established $\left(0^{\circ}, 15^{\circ}, 30^{\circ}\right.$, and $\left.45^{\circ}\right)$. These two independent variables produced a 4 (gap distance) $\times 4$ (orientation difference) factorial design. Participants undertook two experimental blocks, each consisting of 32 trials (a total of 64 trials) presented in a randomized order. Figure 4 displays examples of the experimental conditions in Experiment 2.

\section{Results and Discussion}

The mean proportions of CDDI or CDDS for the full stimulus presentation time $(30 \mathrm{sec})$ for each condition across all participants are shown in Figure 5A. A two-way (gap distance $X$ orientation difference) repeated measures ANOVA was performed for the mean proportions of CDDI and CDDS, respectively. Overviews of the results of ANOVA are presented in Table 2. In the independent MIB mode, the main effects of gap distance and orientation difference were significant. A post hoc analysis with Ryan's method showed that the proportion of CDDI increased as the gap distance between the inner and outer targets increased from 0.2 to $0.8 \mathrm{deg}$ (among all conditions except for that between 0.2 and $0.4 \mathrm{deg}, p<.05$ ). In the main effect of orientation difference, there were also significant differences between the $0^{\circ}$ and $45^{\circ}$ and between the $0^{\circ}$ and $30^{\circ}$ conditions $(p<.05)$. There was also significant interaction between these two factors. The simple main effect analysis revealed the significant effects of the orientation difference for the 0.6 and $0.8 \mathrm{deg}$ conditions of gap distance $[0.6 \mathrm{deg}, F(3,48)=9.94, p<.001 ; 0.8 \mathrm{deg}$, $F(3,48)=11.77, p<.001]$. A post hoc analysis with Ryan's method showed that the proportion of CDDI increased as the orientation difference between the inner and outer targets increased from $0^{\circ}$ to $45^{\circ}(0.6 \mathrm{deg}$, among all conditions except for those between the $0^{\circ}$ and $15^{\circ}$ and between the $30^{\circ}$ and $45^{\circ} ; 0.8 \mathrm{deg}$, between the $0^{\circ}$ and $15^{\circ}$, between the $0^{\circ}$ and $30^{\circ}$, and between the $0^{\circ}$ and $45^{\circ}$ conditions, $p<$ $.05)$. In the simultaneous MIB mode, the main effects of the gap distance and orientation difference were significant in both dependent variables. There was no significant interaction between these two factors. A post hoc analysis with Ryan's method was applied to reveal the differences between the gap distance conditions. The results showed that the proportion of CDDS decreased as the gap distance between the inner and outer targets increased from 0.2 to $0.8 \mathrm{deg}$ (among all conditions except for those between the 0.2 and $0.4 \mathrm{deg}$ and between the 0.6 and $0.8 \mathrm{deg}, p<.05$ ). In the main effect of orientation difference, there were also significant differences between the $0^{\circ}$ and $30^{\circ}$ and between the $0^{\circ}$ and $45^{\circ}$ conditions $(p<.05)$.

A two-way ANOVA (gap distance $\times$ orientation difference) was performed for the mean RDDS percentage data. Figure $5 \mathrm{~B}$ shows the mean proportion of RDDS in the total cumulative MIB duration for each condition, across all the participants. The ANOVA is presented in Table 2, in which it can be seen that the main effects of gap distance and orientation difference were significant. A post hoc analysis with Ryan's method was applied to reveal the differences between the gap distance conditions. The results showed that the proportion of RDDS decreased as the gap distance between the inner and outer targets increased from 0.2 to $0.8 \mathrm{deg}$ (between all pairs of conditions except for

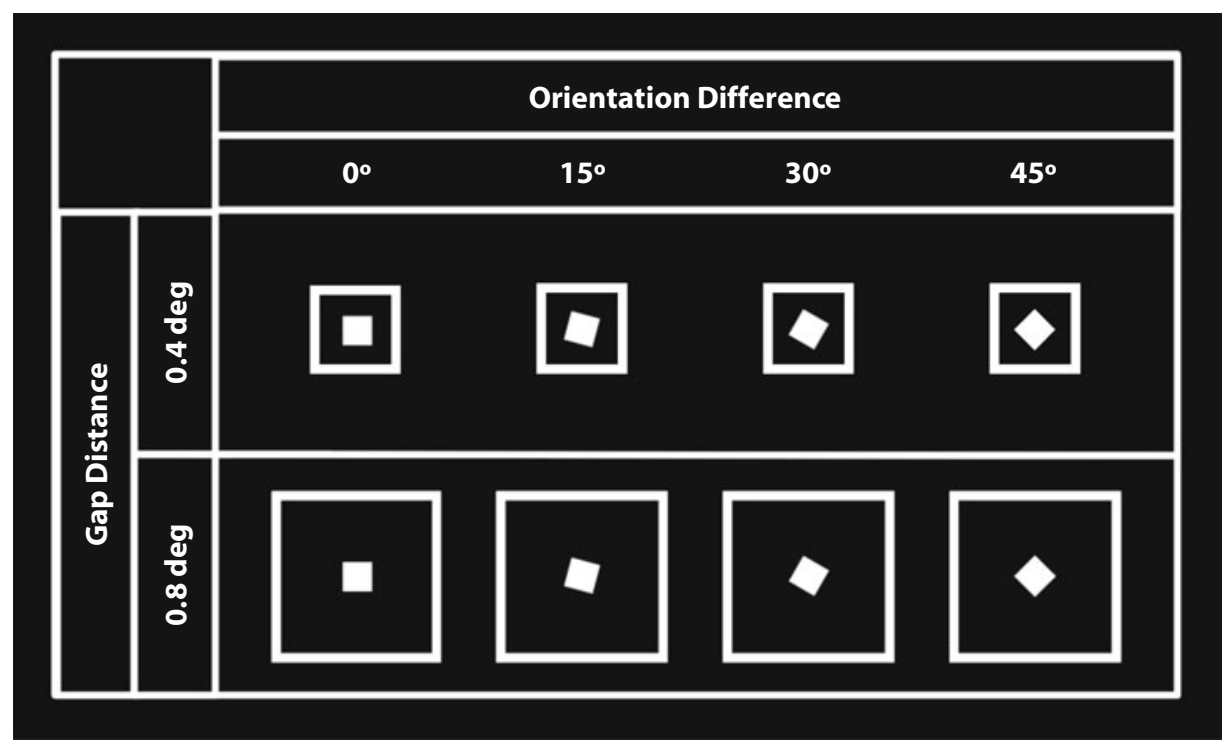

Figure 4. Examples of the visual targets used in Experiment 2. Similar to Experiment 1, the gap distance between the inner and outer squares also ranged from 0.2 to $0.8 \mathrm{deg}$. For each gap distance condition, the position of the inner square was centered, and the inner square was rotated by $0^{\circ}, 15^{\circ}$, $30^{\circ}$, or $45^{\circ}$. 
A

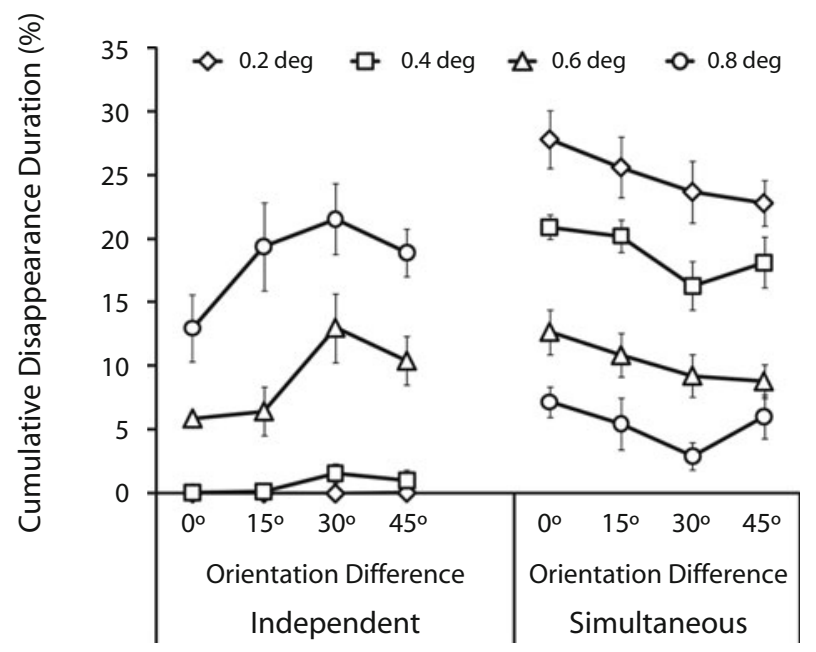

B

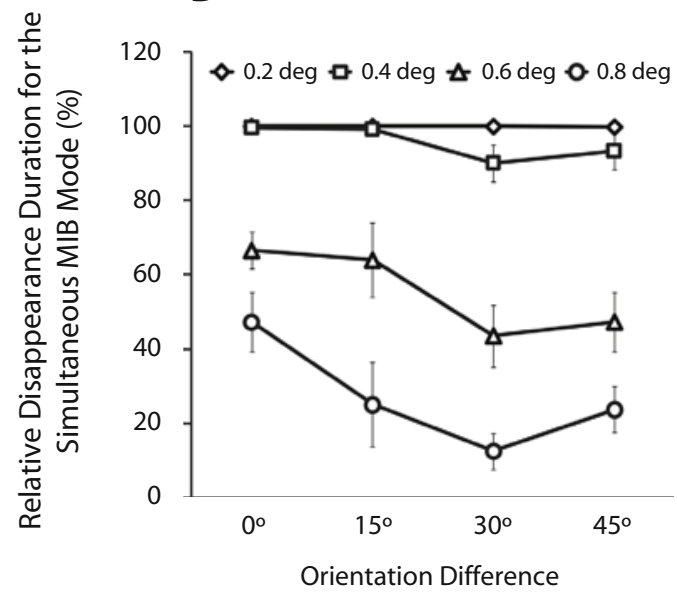

C

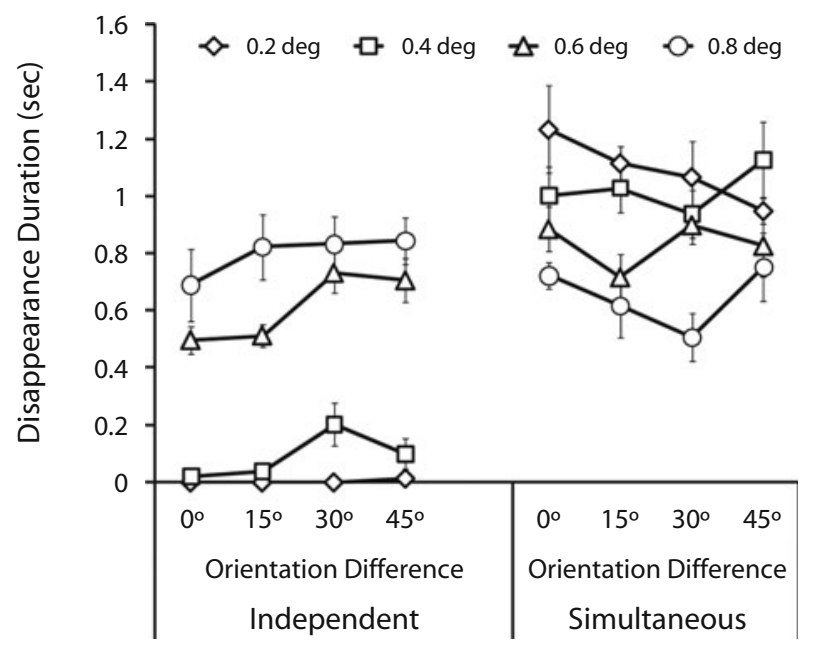

Figure 5. The results of Experiment 2 for each condition. The error bar denotes standard error $(N=5)$. The absence of an error bar means that the SE is too small to be displayed. (A) The $y$-axis indicates the mean proportion of CDDI or proportion of CDDS for the full stimulus presentation time ( $30 \mathrm{sec})$. (B) The $y$-axis indicates the mean proportion of RDDS in the total cumulative MIB. For panels A and $B$, the proportion of RDDS decreased significantly when the orientation difference was relatively high in the 0.6- to 0.8 -deg gap distance conditions. On the other hand, the proportion of CDDI increased as the orientation difference increased in the 0.6- to 0.8-deg gap distance. (C) The $y$-axis indicates the mean disappearance durations of independent or simultaneous MIB modes. For panel C, note that the zero has been used as a missing value. In Experiment 2, the tendency found in the mean disappearance duration was also different from the proportions of CDDI, CDDS, and RDDS.

that between 0.2 and $0.4 \mathrm{deg}, p<.05)$. In the main effect of orientation difference, there were also significant differences between the $0^{\circ}$ and $30^{\circ}$ and between the $0^{\circ}$ and $45^{\circ}$ conditions $(p<.05)$. There was a significant interaction between these two factors. The simple main effect of the orientation difference was significant for the 0.6 and $0.8 \mathrm{deg}$ conditions of gap distance $[0.6 \mathrm{deg}, F(3,48)=$ $9.20, p<.001 ; 0.8 \mathrm{deg}, F(3,48)=14.41, p<.001]$. A post hoc analysis with Ryan's method indicated that the proportion of RDDS decreased as the orientation difference between the inner and outer targets increased from $0^{\circ}$ to $45^{\circ} \mathrm{deg}(0.6$ among all the orientation difference condi- tions except for those between the $0^{\circ}$ and $15^{\circ}$ and $30^{\circ}$ and $45^{\circ}$ conditions, $p<.05$; and $0.8 \mathrm{deg}$, between the $0^{\circ}$ and $15^{\circ}, 0^{\circ}$ and $30^{\circ}$, and $0^{\circ}$ and $45^{\circ}$ conditions, $p<.05$ ).

We performed a two-way (gap distance $\times$ contour closure level) repeated measures ANOVA for the mean disappearance durations of independent or simultaneous MIB modes, respectively. Figure 5C shows the mean disappearance durations of independent or simultaneous MIB modes for each condition across all participants. Overviews of the results of the ANOVA are presented in Table 2. In the independent MIB mode, the main effects of gap distance and orientation difference were sig- 
Table 2

ANOVA Summaries for Each Condition in Experiment 2

\begin{tabular}{|c|c|c|}
\hline Factors & Independent Significance & Simultaneous Significance \\
\hline \multicolumn{3}{|c|}{ Proportions of CDDI and CDDS } \\
\hline Gap distance (GD) & $F(3,12)=30.55, p<.001$ & $F(3,12)=24.07, p<.001$ \\
\hline Orientation difference (OD) & $F(3,12)=10.83, p<.001$ & $F(3,12)=5.75, p<.05$ \\
\hline $\mathrm{GD} \times \mathrm{OD}$ & $F(9,36)=3.63, p<.005$ & $F(9,36)=0.64, p=.760$ \\
\hline \multicolumn{3}{|c|}{ Proportion of RDDS } \\
\hline GD & & $F(3,12)=60.34, p<.001$ \\
\hline OD & & $F(3,12)=16.69, p<.001$ \\
\hline $\mathrm{GD} \times \mathrm{OD}$ & & $F(9,36)=3.35, p<.005$ \\
\hline \multicolumn{3}{|c|}{ Disappearance Duration } \\
\hline GD & $F(3,12)=45.35, p<.001$ & $F(3,12)=6.70, p<.01$ \\
\hline OD & $F(3,12)=15.49, p<.001$ & $F(3,12)=1.05, p=.404$ \\
\hline $\mathrm{GD} \times \mathrm{OD}$ & $F(9,36)=1.82, p=.099$ & $F(9,36)=1.34, p=.251$ \\
\hline
\end{tabular}

nificant, but there was no significant interaction between these two factors. A post hoc analysis with Ryan's method showed that the mean disappearance duration of the independent MIB mode increased as the gap distance between the inner and outer targets increased from 0.2 to $0.8 \mathrm{deg}$ (among all conditions except for those between the 0.2 and $0.4 \mathrm{deg}$ and between the 0.6 and 0.8 ones, $p<.05$ ). In the main effect of orientation difference, there were also significant differences among all conditions except for those between the $0^{\circ}$ and $15^{\circ}$ and between the $30^{\circ}$ and $45^{\circ}$ conditions $(p<.05)$. In the simultaneous MIB mode, the main effect of the gap distance was significant. There was no significant interaction between these two factors. A post hoc analysis with Ryan's method showed that the mean disappearance duration of simultaneous MIB mode decreased as the gap distance between the inner and outer targets increased from 0.2 to $0.8 \mathrm{deg}$ (between the 0.2 and $0.8 \mathrm{deg}$ and between the 0.4 and $0.8 \mathrm{deg}$ conditions, $p<.05)$.

In Experiment 2, we manipulated the gap distance between the targets and their orientation difference in order to investigate how the proximity and orientation similarity cues affect object representation in combination. Similar to those of Experiment 1, the results of Experiment 2 showed that as the gap distance increased from 0.2 to $0.8 \mathrm{deg}$, the proportions of the CDDS and RDDS gradually attenuated, whereas the proportion of CDDI gradually ascended. Furthermore, with a gap distance between the targets of $0.2 \mathrm{deg}$, the proportion of RDDS was the largest, regardless of the orientation difference. In contrast, the proportion of CDDI was the lowest in the $0.2 \mathrm{deg}$ of gap distance, regardless of the orientation difference. In addition, as the orientation difference between the inner and outer targets increased, the proportion of RDDS decreased in the 0.6 to 0.8 deg of gap distance conditions, whereas the proportion of CDDI gradually increased in the 0.6 to $0.8 \mathrm{deg}$ of gap distance conditions in Experiment 2.

Similar to those in Experiment 1, the results found for the mean disappearance duration in Experiment 2 was not always consistent with those for other dependent variables. In Experiment 1, some combined effects of the perceptual grouping cues were obtained on the mean disappearance duration (especially for the simultaneous MIB mode), but there are no combined effects on the mean disappearance duration in Experiment 2. For the mean disappearance duration, Carter and Pettigrew (2003) reported that the strength of perceptual grouping (orientation difference) affected the proportion of the cumulative disappearance duration but did not affect the mean disappearance duration in MIB. This earlier work and our results suggested the possibility that the mean disappearance duration might not be sensitive to the perceptual grouping cues. Overall, the results suggest that the visual system tends to group the targets into a complete object by using orientation similarity cues when visual stimuli are presented with 0.6 or 0.8 deg of gap distance.

\section{GENERAL DISCUSSION}

In this study, we investigated the combined effects of perceptual grouping cues on object representation by using independent or simultaneous disappearances of the target(s) in MIB. The results of the present study revealed that the gap distance between the targets (hereafter, referred to as simply "separation") modulates the availability of contour closure and orientation similarity cues in forming a complete object representation. The results of Experiments 1 and 2 showed that the smaller the separation between targets (ranging from 0.2 to $0.8 \mathrm{deg}$ ), the longer both the inner and outer targets disappeared from visual awareness as complete objects. When the separation between the targets was $0.2 \mathrm{deg}$, the proportions of CDDS or RDDS were the largest, whereas the proportion of CDDI was the lowest regardless of contour closure and orientation similarity cues. The results indicate that a relatively high proximity cue $(0.2 \mathrm{deg}$ separation between the targets) is the most dominant perceptual grouping cue. However, the proximity cue was not always dominant in the formation of a complete object representation. The contour closure cue produced simultaneous disappearance when the separation between the targets ranged from 0.4 to $0.8 \mathrm{deg}$, as did the orientation similarity cue when the separation between the targets was between 0.6 and $0.8 \mathrm{deg}$.

Previous studies found that when some visual targets and distractors were presented with a separation of less 
than $0.5 \mathrm{deg}$, with the targets turning into one group, participants could discriminate the targets from the distractors more rapidly than in conditions with more than 0.5-deg separations (Palmer \& Beck, 2007). As previous studies suggested, the proximity cue is the most robust and fundamental cue for forming object representation (Kubovy et al., 1998; Palmer \& Beck, 2007). Other previous studies using MIB also showed that when targets were presented with a separation of less than $0.5 \mathrm{deg}$, the stimuli simultaneously disappeared and reappeared from visual awareness (Bonneh et al., 2001; Mitroff \& Scholl, 2005). As described above, the effective range of the proximity cues seems to be almost the same in the present and previous studies, whether or not the perceptual disappearance phenomenon was used.

Our results revealed that only the proximity cue affected the independent and simultaneous disappearances of the targets with a separation of $0.2 \mathrm{deg}$, regardless of the closure and similarity cues. The dominance of the proximity cue gradually decreased when the separation was wider than $0.4 \mathrm{deg}$. Instead, contour closure and orientation similarity cues began to affect independent or simultaneous disappearances in conditions involving relatively large separations (for the contour closure cue, $0.4 \mathrm{deg}$ or more; for the orientation similarity cue, 0.6 deg or more) between the targets. The results suggest that perceptual grouping cues differentially contribute to object representation depending on the separation between the targets; that is, the visual system weights each perceptual grouping cue based on the separation between visual stimuli and forms a complete object representation.

Whereas previous studies investigated the effective temporal range of those cues using a microgenetic analysis (Hadad \& Kimchi, 2008; Kimchi, 2000), and the effects of temporal contrast modulation on binocular rivalry (Alais \& Blake, 1999), we investigated the effective spatial range of each perceptual grouping cue in this study. These studies suggested that the effect of the proximity cue is not always dominant over other perceptual grouping cues in forming object representation in the temporal domain. Meanwhile, our study indicates that the effects of perceptual grouping cues on object representation vary depending on the separation between the visual stimuli. However, it remains unclear whether the separation modulates the effective temporal range of each perceptual grouping cue, or vice versa. Future research is needed to investigate this issue in more detail.

\section{Involvement of Visual Attention in MIB}

Several studies suggest that visual attention is directed to perceptual groups (for a review, see Driver, Davis, Russell, Turatto, \& Freeman, 2001) and is involved in the disappearance of targets in MIB. Regarding the former issue, Bonneh et al. (2001) suggested that MIB was similar to clinical cases of attention deficits in which partial invisibility might occur despite the fact that the primary visual areas remain intact (Driver \& Vuilleumier, 2001). Recent studies have manipulated visual attention itself to show that the engagement of visual attention in targets can promote MIB (Carter, Luedeman, Mitroff, \& Nakayama,
2008; Geng, Song, Li, Xu, \& Zhu, 2007; Schölvinck \& Rees, 2009). These findings suggest that there is an involvement of attentional mechanisms in MIB.

Previous studies on visual attention revealed that there are several types of visual attention - spatial, featurebased, and object-based attention (for a review, see Scholl, 2001). In this section, we discuss possible visual processing that influences the occurrence of MIB by exploring the types of visual attention involved in our findings. One might argue that the modulation of the proportions of CDDI, CDDS, and RDDS found in the present study could be mediated by space- or feature-based attention. However, both possibilities are implausible. The spacebased attention theory holds that attention is directed to a region of visual space, is focused at the center of the region, and becomes less effective as the separation from that center is increased (Posner, Snyder, \& Davidson, 1980). This theory suggests that the proportions of CDDI, CDDS, and RDDS simply depend on the separation between the inner and outer targets. However, the results reported here showed that these proportions were affected by the contour closure level when the separation was $0.4 \mathrm{deg}$ or more (Experiment 1), or the orientation difference when the separation increased from 0.6 to $0.8 \mathrm{deg}$ (Experiment 2). Meanwhile, theories of feature-based attention argue that attention is allocated to stimulus features such as color, motion, shape, or orientation rather than to locations (Driver \& Baylis, 1989; Wolfe, Cave, \& Franzel, 1989). According to these theories, if feature-based attention were directed to the shape or orientation of the inner and outer targets, the proportions should vary, depending on the shape or orientation difference between the targets. However, the results showed that the proportions did not vary as a function of shape difference when the separation was $0.2 \mathrm{deg}$ (Experiment 1). Furthermore, the results showed that when the separation ranged from 0.2 to $0.4 \mathrm{deg}$, the proportions were not significantly different in any of the orientation difference conditions (Experiment 2). Therefore, the modulation of the proportions of CDDI, CDDS, and RDDS in the present experiments cannot adequately be explained by the space- or feature-based attention theories. This raises the possibility that the combination of space- and feature-based attention or objectbased attention (directed to a perceptual object per se rather than locations; see Duncan, 1984; Egly, Driver, \& Rafal, 1994) might be involved with the disappearance in MIB. Although there is a need for further consideration of the types of attention that can control the selection of visual inputs to affect visual awareness in MIB, our study implies that attentional selection is supported by a combination of multiple grouping cues.

\section{CONCLUSION}

Experiments using MIB revealed that object representation was supported by a combination of the proximity, contour closure, and orientation similarity cues. The proximity-based grouping has a strong effect on object representation when the separation between visual stimuli is small. Meanwhile, closure and similarity-based group- 
ing starts to affect object representation as the effect of the proximity cues on representation decreases in the case of large separations between visual stimuli. We suggest that the visual system weighs each perceptual grouping cue based on the separation between visual stimuli and forms a complete object representation.

\section{AUTHOR NOTE}

This study was supported by a JSPS Research Fellowship for Young Scientists to Y.K. (No. 195654), and by a MEXT Grant-in-Aid for Scientific Research to J.G. (No. 18330151). This research was conducted at the Department of Psychology, Graduate School of Arts and Letters, Tohoku University. Correspondence concerning this article should be addressed to M. Shibata, Department of Psychology, Tohoku University, 27-1, Kawauchi, Aoba-ku, Sendai, 980-8576, Japan (e-mail: shibatam@, sal.tohoku.ac.jp).

\section{REFERENCES}

Alais, D., \& Blake, R. (1999). Grouping visual features during binocular rivalry. Vision Research, 39, 4341-4353. doi:10.1016/S0042 -6989(99)00146-7

Bonneh, Y. S., Cooperman, A., \& Sagi, D. (2001). Motion-induced blindness in normal observers. Nature, 411, 798-801. doi:10.1038/ 35081073

Caetta, F., Gorea, A., \& Bonneh, Y. (2007). Sensory and decisional factors in motion-induced blindness. Journal of Vision, 7(7, Art. 4), 1-12. doi:10.1167/7.7.4

Carter, O., Luedeman, R., Mitroff, S., \& Nakayama, K. (2008). Motion induced blindness: The more you attend the less you see [Abstract]. Journal of Vision, 8(6), 237a. doi:10.1167/8.6.237

Carter, O. L., \& Pettigrew, J. D. (2003). A common oscillator for perceptual rivalries? Perception, 32, 295-305. doi:10.1068/p3472

DrIVER, J., \& BAYLIS, G. C. (1989). Movement and visual attention: The spotlight metaphor breaks down. Journal of Experimental Psychology: Human Perception \& Performance, 15, 448-456. doi:10.1037/0096 $-1523.15 .3 .448$

Driver, J., Davis, G., Russell, C., Turatto, M., \& Freeman, E. (2001). Segmentation, attention and phenomenal visual objects. Cognition, 80, 61-95. doi:10.1016/S0010-0277(00)00151-7

Driver, J., \& Vuilleumier, P. (2001). Perceptual awareness and its loss in unilateral neglect and extinction. Cognition, 79, 39-88. doi:10.1016/ S0010-0277(00)00124-4

Duncan, J. (1984). Selective attention and the organization of visual information. Journal of Experimental Psychology: General, 113, 501517. doi:10.1037/0096-3445.113.4.501

Egly, R., Driver, J., \& Rafal, R. D. (1994). Shifting visual attention between objects and locations: Evidence from normal and parietal lesion subjects. Journal of Experimental Psychology: General, 123, 161-177. doi:10.1037/0096-3445.123.2.161

ElDER, J. H., \& ZuCKER, S. W. (1993). The effect of contour closure on the rapid discrimination of two-dimensional shapes. Vision Research, 33, 981-991. doi:10.1016/0042-6989(93)90080-G

EldER, J. H., \& ZUCKeR, S. W. (1994). A measure of closure. Vision Research, 34, 3361-3369. doi:10.1016/0042-6989(94)90070-1

Elder, J. H., \& ZuCKer, S. W. (1998). Evidence for boundaryspecific grouping. Vision Research, 38, 143-152. doi:10.1016/S0042 $-6989(97) 00138-7$

Feldman, J. (2003). What is a visual object? Trends in Cognitive Sciences, 7, 252-256. doi:10.1016/S1364-6613(03)00111-6

Funk, A. P., \& PetTigrew, J. D. (2003). Does interhemispheric competition mediate motion-induced blindness? A transcranial magnetic stimulation study. Perception, 32, 1325-1338. doi:10.1068/p5088

GenG, H. Y., Song, Q. L., LI, Y. F., Xu, S., \& ZHU, Y. (2007). Attentional modulation of motion-induced blindness. Chinese Science Bulletin, 52, 1063-1070. doi:10.1007/s11434-007-0178-0

Graf, E. W., Adams, W. J., \& Lages, M. (2002). Modulating motioninduced blindness with depth ordering and surface completion. Vision Research, 42, 2731-2735. doi:10.1016/S0042-6989(02)00390-5
HaDAD, B. S., \& Kimchi, R. (2008). Time course of grouping of shape by perceptual closure: Effects of spatial proximity and collinearity. Perception \& Psychophysics, 70, 818-827. doi:10.3758/PP.70.5.818

Hamburger, K., Prior, H., Sarris, V., \& Spillmann, L. (2006). Filling-in with colour: Different modes of surface completion. Vision Research, 46, 1129-1138. doi:10.1016/j.visres.2005.08.013

Hsu, L. C., Yeh, S. L., \& Kramer, P. (2004). Linking motion-induced blindness to perceptual filling-in. Vision Research, 44, 2857-2866. doi:10.1016/j.visres.2003.10.029

Hsu, L. C., YeH, S. L., \& Kramer, P. (2006). A common mechanism for perceptual filling-in and motion-induced blindness. Vision Research, 46, 1973-1981. doi:10.1016/j.visres.2005.11.004

Kanai, R., \& Kamitani, Y. (2003). Time-locked perceptual fading induced by visual transients. Journal of Cognitive Neuroscience, 15, 664-672. doi:10.1162/jocn.2003.15.5.664

KImCHI, R. (2000). The perceptual organization of visual objects: A microgenetic analysis. Vision Research, 40, 1333-1347. doi:10.1016/ S0042-6989(00)00027-4

Kovács, I., Papathomas, T. V., YAnG, M., \& FehÉr, Á. (1996). When the brain changes its mind: Interocular grouping during binocular rivalry. Proceedings of the National Academy of Sciences, 93, 1550815511

Kubovy, M., Holcombe, A. O., \& Wagemans, J. (1998). On the lawfulness of grouping by proximity. Cognitive Psychology, 35, 71-98. doi:10.1006/cogp.1997.0673

Kubovy, M., \& Wagemans, J. (1995). Grouping by proximity and multistability in dot lattices: A quantitative Gestalt theory. Psychological Science, 6, 225-234. doi:10.1111/j.1467-9280.1995.tb00597.x

MitrofF, S. R., \& Scholl, B. J. (2005). Forming and updating object representations without awareness: Evidence from motion-induced blindness. Vision Research, 45, 961-967. doi:10.1016/j.visres .2004 .09 .044

Moradi, F., \& Shimojo, S. (2004). Suppressive effect of sustained low-contrast adaptation followed by transient high-contrast on peripheral target detection. Vision Research, 44, 449-460. doi:10.1016/j .visres.2003.10.005

Nelson, R., \& Palmer, S. E. (2001). Of holes and wholes: The perception of surrounded regions. Perception, 30, 1213-1226. doi:10.1068/ p3148

Palmer, S. E., \& Beck, D. M. (2007). The repetition discrimination task: An objective method for studying perceptual grouping. Perception \& Psychophysics, 69, 68-78.

Posner, M. I., SNYder, C. R., \& Davidson, B. J. (1980). Attention and the detection of signals. Journal of Experimental Psychology: General, 109, 160-174.

Scholl, B. J. (2001). Objects and attention: The state of the art. Cognition, 80, 1-46. doi:10.1016/S0010-0277(00)00152-9

SchölvincK, M. L., \& ReEs, G. (2009). Attentional influences on the dynamics of motion-induced blindness. Journal of Vision, $\mathbf{9}(1$, Art. 38), 1-9. doi:10.1167/9.1.38

Vergeer, M. L., \& VAN Lier, R. (2007). Grouping effects in flashinduced perceptual fading. Perception, 36, 1036-1042. doi:10.1068/ p5607

WeRTHEIMER, M. (1923). Untersuchungen zur Lehre von der Gestalt: II. Psychologische Forschung, 4, 301-350. [Partial translation in W. D. Ellis (Ed.) (1950). A source book of Gestalt psychology (pp. 71-81). New York: Humanities Press.]

Wolfe, J. M., CAVE, K. R., \& Franzel, S. L. (1989). Guided search: An alternative to the feature integration model for visual search. Journal of Experimental Psychology: Human Perception \& Performance, 15, 419-433. doi:10.1037/0096-1523.15.3.419

\section{NOTE}

1. To avoid confusion, "deg" is used to denote measurements of the visual stimulus size, and the actual degree sign is used to indicate angles within the orientation difference between visual targets.

(Manuscript received January 15, 2008; revision accepted for publication September 28, 2009.) 